\title{
Is the direct observation of electronic coherence in electron transfer reactions possible?
}

\author{
Andreas Lucke, ${ }^{1,2}$ C.H. Mak, ${ }^{1}$ Reinhold Egger,${ }^{2}$ Joachim Ankerhold,${ }^{2,3}$ Juergen Stockburger, ${ }^{1}$ and Hermann \\ Grabert $^{2}$ \\ ${ }^{1}$ Department of Chemistry, University of Southern California, Los Angeles, CA 90089-0482, USA \\ ${ }^{2}$ Fakultät für Physik, Albert-Ludwigs-Universität, Hermann-Herder-Straße 3, D-79104 Freiburg, Germany \\ ${ }^{3}$ Department of Chemistry, Columbia University, New York, NY 10027, USA
}

(Date: October 13, 2018)

The observability of electronic coherence in electron transfer reactions is discussed. We show that under appropriate circumstances large-amplitude oscillations can be found in the electronic occupation probabilities. The initial preparation of the system is of crucial importance for this effect, and we discuss conditions under which experiments detecting electronic coherence should be feasible. The Feynman-Vernon influence functional formalism is extended to examine more general and experimentally relevant initial preparations. Analytical expressions and path integral quantum dynamics simulations were developed to study the effects of various initial preparations on the observability of electronic coherence.

\section{INTRODUCTION}

The role of quantum coherence in the dynamics of molecular systems in the condensed phase is still largely unclear. According to conventional wisdom, quantum coherence should have little influence on condensed phase dynamics because the dephasing time of any coherent process is expected to be very short for systems with any appreciable number of degrees of freedom. The extent to which conventional wisdom is correct depends on many parameters such as the effective mass of the degree of freedom under consideration, its coupling strength to the bath, and the timescale of the bath motions. Therefore, the understanding of quantum coherence in condensed phase molecular systems can be a rather complicated issue.

The experiments of Vos et al. [1] on the photosynthetic reaction center were one of the first in which vibrational coherence was directly observed in an electron transfer (ET) system. Similar experimental observations in other systems have been reported since [2 7]. The most surprising aspect of the discovery is that quantum coherence is directly detectable and is actually preserved on a rather long timescale even in condensed phase systems with ultrafast solvent dynamics. These direct observations of vibrational coherence in ET systems have led to speculations concerning the possible influence coherent quantum dynamics may have on the ET dynamics itself. There have been a number of theoretical studies on this subject 8 12]. However, experiments have so far been unable to establish a clear connection between vibrational coherence and ET rates.

In this paper, we will focus on electronic coherence in ET reactions. Electronic coherence, if present, would have a more profound effect on the ET dynamics than vibrational coherence. In the absence of the environment, an ET system when put into a state represented by a superposition of two or more electronic eigenstates will evolve coherently. This simple picture of electronic coherence becomes immensely muddled when the environment is taken into account, because the coherent electronic motions can be dephased by the nuclear motions in the environment. In fact, in most situations the electronic motions are so strongly coupled to the nuclear motions that the combined system is better described by the vibronic states. In this way the electronic motions associated with the electronic coherence will acquire a larger effective mass, and the crucial experimental question is whether electronic coherence can be observed directly in ultrafast experiments on a timescale fast enough to beat the dephasing time. A recent experimental attempt by Reid et al. [7] to detect electronic coherence in biruthenium mixed-valence compounds has failed to yield any direct evidence because the dephasing timescale seems to be too short even for the 20 fs time resolution of the experiment. Given this negative result, the possibility of a direct experimental detection of electronic coherence may appear unlikely.

The purpose of this paper is to re-examine the theoretical issues surrounding the observability of electronic coherence in ET systems. Contrary to prevailing skepticism, we believe that there is no intrinsic experimental limitation that fundamentally prevents electronic coherence from being detected. We employ simple arguments to show that the major difficulties encountered in the direct experimental observation of electronic coherence are related to how the system is prepared initially in the experiment. By using various methods of nonequilibrium initial preparation, the duration of time within which electronic coherence is preserved as well as the amplitude of the coherent oscillations can be increased dramatically. The validity of these simple ideas have been verified by dynamical path integral simulations. Our analyses suggest that electronic coherence can be most easily observed in adiabatic ET systems in which the electronic coupling matrix element is large compared to the frequency of typical solvent motions. ET systems that satisfy these requirements are often found in mixed-valence compounds [13], which have typical electronic coupling matrix elements of the order of several hundred wavenumbers. 
The organization of the paper is as follows. Section II presents a simple analysis of electronic coherence in an ET experiment. Using this analysis, we study the effects of various preparation methods on the strength and the dephasing time of the electronic coherence in the adiabatic limit. Based on these results, we propose a number of specific ways to engineer the initial preparation of the system so that electronic coherence could be more easily detected. Section III develops these qualitative ideas further by a detailed quantitative analysis using the two-state spin-boson Hamiltonian as a model for mixed-valence compounds. The Feynman-Vernon influence functional method is extended to allow for more general initial preparations, and the concept of an "equivalent external field" is developed. Accurate quantum dynamics simulations show that the qualitative predictions of the simple theories from Section III are generally preserved when the full solvent dynamics is taken into account. For completeness, we also include in Section [IV] a brief summary of results for the nonadiabatic region.

\section{ELECTRONIC COHERENCE FOR ADIABATIC ELECTRON TRANSFER}

\section{A. Standard Preparation}

In the absence of the solvent, if the two electronic states before and after the charge transfer are denoted $|L\rangle$ and $|R\rangle$, the electronic Hamiltonian can be written in the tight-binding approximation as

$$
\begin{aligned}
H_{e}= & -\frac{\hbar \Delta}{2}(|L\rangle\langle R|+| R\rangle\langle L|) \\
& +\frac{\hbar \epsilon}{2}(|L\rangle\langle L|-| R\rangle\langle R|),
\end{aligned}
$$

where $\epsilon$ is a bias related to the intrinsic difference in the redox free energies $\Delta G^{0}$ of the two electronic states. In condensed phase ET systems, this electronic system is immersed in a bath. In most bimetallic mixedvalence compounds, the electronic coupling matrix elements $\hbar \Delta / 2$ are large enough for them to be classified as adiabatic ET systems [13]. This means that the timescale of the pure electronic motions, set by the electronic matrix element $\hbar \Delta / 2$, is much shorter than the timescale of the bath motions. Although the frequencies of bath motions often cover a broad and almost continuous spectrum, there is often a typical bath frequency $\omega_{c}$ such that motions with frequencies significantly higher than $\omega_{c}$ are absent in the bath 15]. The adiabatic regime is then defined by the condition $\Delta \gg \omega_{c}$.

Under the condition $\Delta \gg \omega_{c}$, we can invoke the BornOppenheimer approximation and arrive at the adiabatic limit of Marcus theory 17 19. In this limit, the bath polarization $\mathcal{E}$ can be treated as a classical coordinate, which couples to the dipole moment of the electronic state. The electronic Hamiltonian is modified by this coupling and it becomes

$$
\begin{aligned}
H_{e}(\mathcal{E})= & -\frac{\hbar \Delta}{2}(|L\rangle\langle R|+| R\rangle\langle L|) \\
& +\left(\frac{\hbar \epsilon}{2}+\mu \mathcal{E}\right)(|L\rangle\langle L|-| R\rangle\langle R|),
\end{aligned}
$$

where $\mu$ is one-half the difference in the dipole moments of the two electronic states. In the adiabatic limit, the bath polarization is slow, so that the Born-Oppenheimer approximation gives as a function of the bath polarization two electronic eigenstates with energies

$$
E_{ \pm}(\mathcal{E})= \pm \sqrt{\left(\frac{\hbar \Delta}{2}\right)^{2}+\left(\frac{\hbar \epsilon}{2}+\mu \mathcal{E}\right)^{2}}
$$

Figure 1 (a) shows the energies of the two eigenstates as a function of the bath polarization $\mathcal{E}$ for a symmetric system $(\epsilon=0)$.

Within Marcus theory the bath polarization obeys linear response. Therefore, the orientation of the bath polarization when the bath is uncoupled to the electronic system has an intrinsic gaussian distribution

$$
\rho_{0}(\mathcal{E})=Z_{0}^{-1} \exp \left(-\beta \mu^{2} \mathcal{E}^{2} / \lambda\right),
$$

where $\beta=1 / k_{B} T, \quad Z_{0}=\int d \mathcal{E} \exp \left(-\beta \mu^{2} \mathcal{E}^{2} / \lambda\right)=$ $\sqrt{\pi \lambda / \mu^{2} \beta}$, and $\lambda$ is the classical reorganization energy.

In a normal ET experiment, we imagine an initial preparation in which we hold the electron in state $|L\rangle$ and thermalize the bath polarization to it. In other words, the bath is prepared initially with a distribution

$$
\rho_{\mathrm{S}}(\mathcal{E})=Z_{\mathrm{S}}^{-1} \exp \left[-\beta\left(\mu^{2} \mathcal{E}^{2} / \lambda+\mu \mathcal{E}\right)\right] .
$$

Related to the intrinsic bath polarization distribution (4), this distribution has the same gaussian shape, but its maximum is shifted to $\mu \mathcal{E}=-\lambda / 2$. This is the usual initial preparation one considers in theoretical descriptions of ET. Here, we shall always refer to it as the "standard preparation".

At time $t=0$, we release the electron and observe its dynamics. Because the bath polarization is slow compared to the electron, we can compute the early-time dynamics of the electron assuming that the bath is stationary. This corresponds to solving for the dynamics of the electron using the two eigenstates (3) for every position of the bath polarization $\mathcal{E}$, and then averaging the result over the distribution function $\rho_{\mathrm{S}}(\mathcal{E})$ in Eq. (5). This procedure is illustrated schematically in Fig. 1(a). Due to the gaussian preparation $\rho_{\mathrm{S}}$, only the polarizations between the two dashed lines are significantly populated. For each $\mathcal{E}$, the dynamics of a biased but free two-level system can be solved giving $P_{L}(t ; \mathcal{E})$, the transient population on the $L$-state, with the initial condition set to $P_{L}(0 ; \mathcal{E})=1$. An average over $\mathcal{E}$ finally produces the observed $P_{L}(t)$ : 


$$
P_{L}(t)=\int_{-\infty}^{\infty} d \mathcal{E} \rho_{\mathrm{S}}(\mathcal{E}) P_{L}(t ; \mathcal{E}) .
$$

The solid line in Fig. 2 illustrates a typical result of Eq. (6) for a symmetric system, $\epsilon=0$. (In Fig. 2, time is measured in units of an inverse frequency, $1 / \omega_{c}$, which for now has no special meaning other than setting a timescale.) As expected for the case of a standard preparation, the $L$-state population remains approximately unity, indicating a very slow ET rate from the $L$ - to the $R$-state. In Section IIIA, we will verify that the qualitative behavior predicted by this adiabatic calculation is indeed correct using a series of exact quantum simulations.

\section{B. Nonstandard Preparations}

In this section, we will examine several experiments in which the initial preparation of the bath is carried out by a distribution function different from $\rho_{\mathrm{S}}$. We call these "nonstandard preparations". In these experiments, the electron is held in the $L$-state for time $t<0$ as in the standard experiment, but the bath is prepared by a generalized distribution function

$$
\rho_{\mathrm{NS}}(\mathcal{E} ; \bar{q}, \bar{\beta})=Z_{\mathrm{NS}}^{-1} \exp \left[-\bar{\beta}\left(\mu^{2} \mathcal{E}^{2} / \lambda-\bar{q} \mathcal{E} \mu\right)\right],
$$

in which the bath polarization $\mathcal{E}$ is equilibrated to an effective electronic dipole moment of magnitude $\bar{q} \mu$ instead of $\bar{q}=-1$ as in $\rho_{\mathrm{S}}$. In addition, the initial bath polarization distribution can have a different width, i.e. be at an effective temperature $\bar{\beta}$.

Within Marcus theory, the initial bath preparation specified by $\rho_{\text {NS }}$ represents the proper description for systems which are not necessarily thermalized with the bath coupled to the electron held fixed in the $L$-state. The distribution in Eq. (7) differs from that in Eq. (5) by a horizontal shift in $\mu \mathcal{E}$ by an amount of $(\bar{q}+1) \lambda / 2$. If $\bar{\beta} \neq \beta$, the distributions also have a different width. The following discussion focuses predominantly on the effect of the position of the distribution only, not its shape. Therefore, we will concentrate on those nonstandard distributions with $\bar{\beta}=\beta$ and refer to them specifically as "shifted bath preparations". In the following, we suggest several possible scenarios which give rise to an initial preparation described by Eq. (7). Similar nonstandard initial preparations have been considered in Refs. [11, 12] for the nonadiabatic regime, but to our knowledge nonstandard initial preparations have not been studied for the adiabatic regime before.

First, consider the experiment depicted in Fig. 11(b). Using a symmetric Fe(III)-Fe(III) compound as an example, we imagine an experiment in which an extra electron is photoinjected into the ion on the left at time $t=0$. Prior to the injection, the electronic state has a zero dipole moment, so the bath is initially prepared with a distribution function $\rho_{\mathrm{NS}}$ characterized by $\bar{q}=0$ and $\bar{\beta}=\beta$. Using the adiabatic approximation described in Sect. II A, we can now compute the transient electronic population on the left ion $P_{L}(t)$ using $\rho_{\mathrm{NS}}$ for the bath preparation. The result is shown in Fig. 2 as the shortdashed line. Remarkably, $P_{L}(t)$ shows large-amplitude coherent oscillations. The origin of this coherent behavior is purely electronic, and it can be explained by referring to the inset in Fig. If(b). The relevant range of $\mathcal{E}$, depicted by the region between the dashed lines, is now shifted to near $\mathcal{E}=0$. Inside this range, the two adiabatic electronic eigenstates are close in energy (approximately $\Delta$ apart). Consequently, when forced into the $L$-state which is a superposition of the energy eigenstates, the electronic motion will exhibit a coherent oscillation with a frequency that is of the order of $\Delta$.

At this point, it is necessary to examine why preparing the bath thermally at $\bar{q}=-1$ does not seem to yield similar coherent oscillations, whereas the nonstandard preparation does. The absence of oscillations in the first case is not due to dephasing, because so far the bath has been assumed to be static and hence nondissipative. There are no oscillations, simply because for polarizations $\mathcal{E}$ relevant for this initial preparation (provided one has similar parameters as in Fig. 2), each of the electronic states $|L\rangle$ and $|R\rangle$ is close to one of the two energy eigenstates, $\left|E_{-}\right\rangle$and $\left|E_{+}\right\rangle$, respectively, which themselves are of course stationary. On the other hand, if $\mathcal{E}=0$, both $|L\rangle$ and $|R\rangle$ are superpositions of both energy eigenstates to the same extent. Therefore, when the initial preparation provides polarizations close to zero, we can expect large oscillations in the time-dependent electronic occupation probabilities.

Though elementary, this observation is the conceptual foundation of the present work. We see that in a usual experiment (i.e. with the standard preparation), lack of electronic oscillations is expected for reasons that have nothing to do with dephasing. The conventional wisdom that absence of electronic oscillations is due to rapid dephasing is not necessarily correct, because coherent oscillations can be suppressed by the way the system is prepared instead of by dephasing. Moreover, the standard way ET experiments are carried out are not the proper experiments for observing electronic coherence. We want to re-examine this issue in detail. From our analysis of the adiabatic limit we can understand the physical picture and try to construct experiments which are best positioned to detect electronic coherence directly. In Section III we go beyond the adiabatic limit and formulate the problem rigorously including the quantum dynamics of the bath as well as shifts in the initial preparations. With that formulation, we will verify that even for moderately large $\Delta / \omega_{c}$, the adiabatic predictions remain qualitatively correct.

We can imagine a few more experimental situations in which nonstandard initial preparations arise. In the experiments by Vos et al. [1], for example, the donor state is populated by a fast laser pulse which leaves the environment essentially unchanged during excitation. Therefore, 
the initial bath polarization distribution corresponds to a gaussian wavepacket that is equilibrated with respect to an electronic state different from $|L\rangle$. Such an initial preparation can be characterized by a $\rho_{\mathrm{NS}}$ with a $\bar{q} \neq-1$. In Fig. 2, we have also shown $P_{L}(t)$ for a nonstandard preparation with $\bar{q}=-0.5$. This could be achieved, say, by ultrafast laser pulse excitation from a ground state with an electronic dipole moment of magnitude $-0.5 \mu$. Even for this situation, we find that $P_{L}(t)$ (long-dashed curve in Fig. 2) still displays some, albeit much weaker, coherent oscillations.

Another experiment in which a nonstandard bath preparation can be achieved is an ultrafast back electron transfer (b-ET) experiment depicted in Fig. 1 (c). In this experiment, a fast laser pulse is used to excite a mixedvalence compound into the charge-transfer (CT) band to initiate a fast b-ET reaction back to the ground state. When the system is placed on the CT state, the polarization will quickly move away from the excitation region and may not have sufficient time to equilibrate. This situation is describable by a nonstandard bath preparation $\rho_{\mathrm{NS}}$ with $\bar{q}=+1$.

The few experiments described above may not always be practically feasible. For example, in the experiment illustrated in Fig 11(a), only in a highly idealized situation will the photoinjected electron accquire a pure $|L\rangle$ state instead of a superposition of $|L\rangle$ and $|R\rangle$. There is no doubt that the proper initial preparation will be tricky to establish, otherwise electronic coherence would have been seen already. Alternative ways to achieve similar shifted preparations will need to be formulated.

In Sect. IIIB, it is shown that remarkably the same ET behavior associated with shifted preparations of the type of $\rho_{\mathrm{NS}}$ in Eq. (7) can also be achieved by using the standard preparation, but coupling the electron to a suitably chosen external time-dependent electric field that is tailored to mimic the effects of the shifted bath. For example, instead of releasing the electron into a polarized environment, one could imagine an initially unpolarized bath and apply an external field that couples to the electric dipole moment just as the bath polarization would, thus producing identical ET behavior. As the effects of the initial bath preparation dissipates, we would have to adjust the external field to mimic the time-dependent effects of the bath. We call such a field the "equivalent external field", because it exactly compensates for the effects on the ET that a different initial preparation would have had. This method is depicted schematically in Fig. 11(d). A time-dependent electric field like the one depicted in Fig. $\mathbb{1}(\mathrm{d})$ is difficult to generate experimentally, because the decay time of the field must be similar to the bath relaxation time which is often of the order of a few hundred femtoseconds. An electric field of sufficient strength that varies on that timescale can probably only be generated optically. We will show in Section III, that approximating the true equivalent electric field crudely by a static field plus a CW laser field and applying it to the equilibrium system can induce electronic coherent behaviors very similar to that with a $\bar{q}=0$ initial preparation. Such a field is schematically shown in Fig. 11(e).

Finally, there may be additional difficulties with the experimental observability of electronic coherence. Even if the proper initial preparations or equivalent electric fields can be achieved, the coherent oscillations in the electronic populations may be difficult to detect experimentally. Since the coherent oscialltaions, if present, are expected to have a frequency $\sim \Delta$, given the best time resolution of currently available lasers of approximately $20 \mathrm{fs}$, this limits the systems in which coherent ET dynamics can be observed to those having $\Delta$ smaller than a few hundred wavenumbers.

\section{THEORY: PATH INTEGRAL FORMULATION AND QUANTUM MONTE CARLO SIMULATIONS}

\section{A. Nonstandard Initial Preparations}

In this section, we want to verify that the qualitative predictions from the last section concerning the detectability of electronic coherence are still correct when the full quantum dynamics of the bath are taken into account.

ET with a quantum mechanical bath has thoroughly been studied theoretically in the framework of the spinboson model 14,19,21. In the spin-boson model, the electronic part of the Hamiltonian is described by Eq. (2), but the bath polarization $\mu \mathcal{E}=\sum_{k} c_{k} x_{k}$ is represented by a linear combination of an infinite collection of linearly-responding effective solvent modes $\left\{x_{k}\right\}$ [19]. An infinite number of solvent modes is required for a proper description of a truly dissipative environment. The coupling constants $c_{k}$ that determine the interaction strength of the solvent modes with the electronic states are specified by a spectral density $J(\omega)$ [21].

Within this model, the ET dynamics, that is the time dependent population probabilities $P_{L}(t)\left(P_{R}(t)\right)$ of the left (right) state can be determined numerically exactly using a method known as the quantum Monte Carlo (QMC) path integral method. This technique has been successfully employed to investigate the dynamics in many ET systems and has been described at length in many of our previous papers, e.g. Refs. [33 35], and reviews [36,37. We will not repeat the details here.

We imagine an ET experiment in which the electron is created in the donor state prior to the start of the reaction. If the electron is held in the donor state for a period of time long enough for the bath to equilibrate to it, the initial density matrix is given by a "factorized" form 14.21:

$$
\rho(0)=|L\rangle\langle L| e^{-\beta\left(H_{B}+\mu \mathcal{E}\right)},
$$

where $H_{B}$ is the bare bath Hamiltonian. In typical situations, the electron is strongly solvated in the donor state, 
and the initial preparation specified by Eq. (8) represents the standard way an ET experiment is often carried out [26. In the classical limit, this is equivalent to the standard preparation we considered in Sect. II A and depicted in Fig. 11(a).

Nonstandard initial conditions result when the bath does not have sufficient time to equilibrate to the electron created in the donor state. An example of an experiment in which this may occur is the one depicted in Fig. 1(b) and considered in Sect. II B. Following the photoinjection of an electron into the donor state in Fig. 1(b), the bath may not have enough time to equilibrate to the electron if the subsequent ET rate is rapid. This would result in a factorized initial condition similar to Eq. (8), except the bath is equilibrated to a zero-dipole-moment electronic system such that the preparation is now defined by the initial density matrix

$$
\rho(0)=|L\rangle\langle L| e^{-\beta H_{B}} .
$$

This gives rise to the nonstandard shifted bath preparation we have considered in Sect. II B. In general, we can represent any factorized system preparation by an initial density matrix in the form

$$
\rho(0 ; \bar{q})=|L\rangle\langle L| e^{-\beta\left(H_{B}-\bar{q} \mu \mathcal{E}\right)},
$$

where the parameter $\bar{q}$ determines whether the bath preparation is standard or shifted. Obviously, $\bar{q}=-1$ corresponds to the standard preparation and $\bar{q}=0$ to the experiment of Fig. 1 (b). The same $\rho(0)$ can also be used to describe the initial preparation in the b-ET experiment in Fig. 11(c), the appropriate value of $\bar{q}$ there being +1 . For a laser pulse excitation from the groundstate Born-Oppenheimer surface, at least in principle, any value of $\bar{q}$ can be generated.

The computer programs from the previous QMC studies 33 37] were modified to include the nonstandard preparation defined by Eq. (10). A series of QMC simulations were performed for a number of ET parameters and different nonstandard preparations to examine the influence of shifted bath preparations on the time-dependent occupation probability $P_{L}(t)$. In particular, we looked for conditions under which large-amplitude oscillations were evident in $P_{L}(t)$.

Since the bath obeys linear response, all effects of the bath on the electronic states can be described by the bath polarization correlation function, which is related to the spectral density $J(\omega)$ by

$$
\langle\delta \mathcal{E}(t) \delta \mathcal{E}(0)\rangle=\frac{\hbar}{(2 \mu)^{2} \pi} \int_{0}^{\infty} d \omega J(\omega) \frac{\cosh (\omega[\hbar \beta / 2-i t])}{\sinh (\omega \hbar \beta / 2)} .
$$

For most ET systems, a sufficiently realistic choice for $J(\omega)$ is the ohmic spectral density with an exponential cutoff,

$$
J(\omega)=(2 \pi \hbar \alpha) \omega e^{-\omega / \omega_{c}}
$$

where the dimensionless friction parameter $\alpha$ is related to the classical reorganization energy $\lambda$ by $\lambda=2 \alpha \hbar \omega_{c}$. A solvent with an ohmic spectral density has a bath polarization correlation function that is approximately constant at short times and (at low temperatures) decays algebraically at long times. This behavior is qualitatively similar to what has been observed in experiments for several solvents [22] and in molecular dynamics simulations 23 25. A bath with an ohmic spectral density describes a polarization that by itself is overdamped and consequently does not intrinsically exhibit vibrational coherence. This ensures that oscillations in the electronic populations whenever detected must be due solely to electronic coherence, but not due to vibrational coherence of the nuclear coordinates.

It is noteworthy that the same ohmic-like spectral density has been found by simulations to characterize the primary ET in bacterial photosynthesis [24]. Therefore, regarding the primary charge separation ET process, vibrational coherence is expected also to have no relevance.

We shall now compare QMC data to the predictions from Sect. II to verify that the general conclusions reached there remain correct when a fully dynamical and dissipative bath is included in the model. First, we examine QMC results for a symmetric $(\epsilon=0)$ ET system with standard and shifted bath preparations. All frequency and energy parameters can be expressed in dimensionless units of $\omega_{c}$, and for this calculation, we have selected $\Delta / \omega_{c}=4, \beta \hbar \omega_{c}=3$ and a friction constant $\alpha$ such that the classical reorganization energy is $\lambda / \hbar \omega_{c}=20$. The parameters are representative of many mixed-valence compounds, which generally have $\omega_{c}$ of the order of a hundred to several hundred $\mathrm{cm}^{-1}$. The QMC results are shown in Fig. 3. For a typical $\omega_{c}=100 \mathrm{~cm}^{-1}$, one $\omega_{c} t$ on the time-axis corresponds to $53 \mathrm{fs}$ in real time.

The solid line in Fig. 3(a) shows QMC data for the standard bath preparation $\bar{q}=-1$. As expected, because the temperature and the reorganization energy chosen put the system in the activated region, the ET rate is slow and the time-dependent occupation probability $P_{L}(t)$ decays so slowly that on the timescale plotted it appears to remain almost constant except for a fast initial transient. The qualitative prediction made in Sect. II A for the same set of parameters is also shown in the same figure as the dashed line. Obviously, the adiabatic theory prediction captures most of the qualitative features of the early-time dynamics of the ET.

Figure 3 (c) shows QMC results for a nonstandard bath preparation with $\bar{q}=0$. This corresponds to a bath initially equilibrated to a zero-dipole-moment electronic state, such as the experiment depicted in Fig. 1(b). In this case, there are large-amplitude oscillations in the electronic population, indicating clearly the presence of electronic coherence. The close resemblance of the exact QMC results to the qualitative adiabatic theory prediction for the same parameters (dashed line) indicates that the reasoning given in Sect. II B for the detectability of electronic coherence in experiments with nonstandard 
bath preparations is indeed correct. Figure 3(b) shows results for an intermediate value of $\bar{q}=-0.5$. Coherent oscillations are still present, though to a smaller extent.

The discrepancies between QMC data and the adiabatic theory predictions visible in Fig. 3 are as expected. Since the adiabatic limit does not have dissipation, the electronic coherence persists indefinitely there, whereas the oscillations observed in the QMC data disappear rather quickly. Consequently, an exact QMC calculation is necessary to estimate whether the coherence predicted by qualitative adiabatic theory is indeed preserved for a long enough period of time so that oscillations can be detected in experiments. Another discrepancy between QMC and adiabatic prediction is that the oscillation frequency in the QMC results is initially similar to the adiabatic prediction, but then increases with time. This behavior is also easily rationalized with the picture of a dynamical polarization field which moves away from the barrier region with time into regions where the difference between the energy eigenvalues is larger, resulting in higher oscillation frequencies.

We have shown results for only one set of parameters. A large number of QMC calculations on other parameter sets have also been performed, and we found that similar coherence effects can be observed for many other systems as well, as long as $\Delta$ is similar to or larger than $\omega_{c}$. Our goal is to highlight the possible electronic coherence effects that could be detected in experiments, but not to give a detailed range of parameters within which electronic coherence can be observed (such a task would have been overwhelming). Therefore, we will not explicitly show results from the other calculations here.

Results presented so far apply to symmetric systems. The qualitative behavior can change quite dramatically depending on the bias $\epsilon$. Figure 1 shows effects of standard and nonstandard bath preparation in the activationless region where $\hbar \epsilon=\lambda$. In the activationless case, if the bath was completely static, even a standard preparation would place the bath inside a region where the two energy eigenstates are close to each other, and adiabatic theory would therefore predict large coherent oscillations. Clearly the QMC data in Fig. 1 for $\bar{q}=-1$ show no such oscillations. Thus, adiabatic theory delivers a qualitatively incorrect picture for this situation. The reason why adiabatic theory is wrong is not difficult to understand. When the bath is placed right at the activationless crossing point on the Marcus parabola where there is no thermal barrier to cross from the donor to the acceptor state, the bath should move rapidly to form the product. The adiabatic assumption that the bath is slow in the activationless region (and regions close to it) is incorrect, rendering the qualitative prediction of adiabatic theory invalid. In reality, the bath being dynamical moves quickly away from the crossing region into regions where the difference between the energy eigenvalues is much larger, thus destroying any sign of coherence.

Next, we consider QMC results for the b-ET experiment depicted in Fig. 1 1 (c). The physical situation is the following. For all $t<0$, the electron is held fixed in the $|R\rangle$ state. Then, at $t=0$, the electron is excited to the $|L\rangle$ state by an ultrafast laser pulse, such that the position of the environment remains essentially unchanged, i.e. with the nonstandard preparation characterized by $\bar{q}=+1$. Figure 5 shows results for a b-ET experiment with the same parameters as in Fig. 3, except a nonstandard bath preparation of $\bar{q}=+1$ was used. Once again, coherent electronic oscillations are clearly observable. Although the electronic coherence is evident in Fig. 5 , its origin is more subtle than the results exhibited in Fig. 3 and this deserves a closer examination.

When the system is excited to the $|L\rangle$ state, the bath being dynamical will move down the Marcus parabola toward the crossing region. For this reason, the predictions of adiabatic theory, which assumes the bath is completely static, are invalid just as in the activationless case. To understand the origin of the coherence, a dynamical picture is required. We focus on the sequence of events following the vertical excitation of the system into the $|L\rangle$ state. As the bath moves toward the crossing region, the electron being coupled to the bath will also evolve. There are two different scenarios. If the bath moves slowly, the electron will have time to adjust to the bath's position and quickly attain a mixture of the CT state and the ground electronic state to approach one of the two energy eigenstates. In this case, the system would exhibit no electronic coherence, because the electron is already in an energy eigenstate. On the other hand, if the bath moves rapidly, the electron may not have time to adjust and would stay in the $|L\rangle$ state, which is not an energy eigenstate. After a brief time delay, which is evident in the QMC data in Fig. . 5, the bath polarization arrives at the crossing region where the two electronic energy eigenstate are closest in energy, and the electronic population would then exhibit large-amplitude coherent oscillations. In other words, whether electronic coherent oscillations are detectable in the electronic occupation probability depends crucially on the electronic coupling $\Delta$ and the bath dynamics timescale $1 / \omega_{c}$. We are in the best position to observe electronic coherence in b-ET experiments if $\Delta$ is larger than or comparable to $\omega_{c}$.

\section{B. The Equivalent Electric Field}

Within the spin-boson model, the path integral formulation easily reveals that the effects of the shifted initial preparations on the ET equal those of an appropriately chosen time-dependent external field. The reasoning goes as follows: When considering an initial preparation as in EQ. (10), the time evolution of the density matrix to a time $t$ is just a special case of the expressions given in 31,21, in that the electronic variable is kept fixed at value $\bar{q}$ throughout the imaginary time path. This leads to an influence functional, in which the shift of the preparation only appears in a term $i(1+\bar{q}) \int_{0}^{t} d \tau R(\tau) \dot{\chi}(\tau)$. 
Here, $\chi=q-q^{\prime}$ and $q, q^{\prime}$ are the electronic variables on the forward and backward paths respectively. $R$ is the imaginary part of a function $Q(z)$, which is $(2 \mu / \hbar)^{2}$ times double integral of the correlation function Eq. (11) with $Q(0)=0$.

In particular,

$$
\begin{aligned}
Q(z) & \equiv \frac{1}{\pi \hbar} \int_{0}^{\infty} d \omega \frac{J(\omega)}{\omega^{2}} \\
& \times \frac{\cosh (\hbar \beta \omega / 2)-\cosh (\omega[(\hbar \beta / 2)-i z])}{\sinh (\hbar \beta \omega / 2)} .
\end{aligned}
$$

Integrating by parts and noting that $\chi(0)=\chi(t)=0$ [the electron is assumed to be in the $|L\rangle$ state initially, and the population in state $|L\rangle$ is measured at time $t]$, this term becomes $-i(1+\bar{q}) \int_{0}^{\tau} d \tau \dot{R}(\tau) \chi(\tau)$. For a strict ohmic spectral density $\left(\omega_{c} \rightarrow \infty\right)$, which is a good approximation for dissipative environments most often encountered in solid-state systems, the integral vanishes and not surprisingly the preparation has no effect. This is why this kind of term is usually tacitly omitted in connection with NIBA calculations 14,21. We will see, however, that for parameters that are typical for many chemical systems, this term does have a significant effect on the ET, even in regimes where the NIBA is still valid, see Sect. IV. From the Marcus picture it is clear that the prefactor, $1+\bar{q}$, is proportional to the displacement of the polarization from the standard preparation that we defined in Section IIA. If $\bar{q}=-1$, the bath is equilibrated to the donor state $|L\rangle$, i.e. the bath is prepared in the standard fashion. In this situation the term above vanishes exactly. It is very advantageous for computations that the influence functional, although reflecting certain initial preparations, does not include integrations over imaginary-time paths.

For a physical understanding, it is helpful to note that a preparation with $\bar{q}$ gives each path a weight $\exp \left[i(1+\bar{q}) \int_{0}^{t} d \tau \dot{R}(\tau) \chi(\tau)\right]$. On the other hand, the action of the bare TLS includes a factor $\exp \left[i \int_{0}^{t} d \tau \epsilon \chi(\tau)\right]$. Obviously, $(1+\bar{q}) \dot{R}(\tau)$ acts just like a time-dependent bias that could be due to a strong electric field coupling to the dipole moment associated with the electronic coordinate. Studying the ET with a nonstandard initial preparation is therefore manifestly equivalent to studying the ET of the same system with a standard initial bath preparation $(\bar{q}=-1)$, but with a time-dependent external field that mimics the preparation effects. We call this field the "equivalent electric field". The physical reason for this observation is that for the ET the source of the field coupling to the electronic dipole moment is unimportant, be it due to the polarization of the solvent or to the external field or both.

The specific form of the equivalent external field can be seen by noting that the phase associated with each electronic path is unchanged under the transformation

$$
(\epsilon, \bar{q}) \rightarrow(\epsilon+(1+\bar{q}) \dot{R}(\tau),-1)
$$

Therefore, the equivalent external field appears as an additional term to the Hamiltonian,

$$
\Delta H=\frac{\hbar}{2}(1+\bar{q}) \dot{R}(t)(|L\rangle\langle L|-| R\rangle\langle R|) .
$$

Eq. (15) establishes the connection between nonstandard initial preparations and the work already done on driven spin-boson systems, see Refs. 38 41]. It is interesting to note that Eq. (15) decreases in time just like the classical damping kernel $\gamma(t)$ [21, since from Eq. (13)

$$
\gamma(t)=(2 / \pi) \int_{0}^{\infty} d \omega \frac{J(\omega)}{\omega} \cos (\omega t)=2 \hbar \dot{R}(t) .
$$

So far, the equivalent external field has just been a name for the effect that nonstandard distributions have on the influence functional. However, as mentioned in Sect. IIB, the equivalent external field may actually be applied to the system to mimic a nonstandard initial preparation in an experiment in which the actual preparation is the standard one, as in Figs. 1 $1(d)$ and (e). To give a flavor, we want to repeat the calculation in Fig. 3 for the standard preparation but under the influence of an equivalent external field. The proper form of the field is given by Eqs. (13) and (15) and shown in the inset of Fig. 6 as the dashed line. In a real experiment, it is much easier to generate a sinusoidal field. Therefore, in the QMC calculation, we used a simple cosine field plus a static bias shown as the solid line in the inset of Fig. 6. With this approximate equivalent electric field, we see from the solid line in Fig. 6 that the external field induces a behavior very similar to the $\bar{q}=0$ initial preparation.

Instead of applying the cosine external field one might try something even simpler and couple a CW source into the system where the phase at $t=0$ is selected at random. The long-dashed curve in Fig. 6 depicts the average of the electronic population from four different calculations, in which the external fields all had a cosine timedependent modulation but had phases shifted by $0,1 / 4$, $1 / 2$ and $3 / 4$ of a period. Although much weaker, coherent oscillations are still clearly evident. A typical field strength required would be about $10^{4} \mathrm{KV} / \mathrm{cm}$, which is not difficult to achieve.

What we have shown up to this point is that given specific initial preparations, electronic coherence can lead to oscillations in the electronic populations that are pronounced enough to be detected in experiments. However, a femtosecond time-resolved spectroscopic analysis of the electronic populations is not a trivial experiment and the interpretation of the signal is often complicated by effects unrelated to the ET itself. Moreover, in some systems where we would expect the ET to be coherent, the oscillations are on the timescale of tens of fs, and therefore cannot be resolved even in the fastest pumpprobe experiments possible today. Finally, we want to point out that our calculations are based on the assumption of a gaussian bath, neglecting e.g. the role of strong 
anharmonic modes that couple to the ET. Clearly, nature is more complicated than our model, but it is safe to conclude that strong dissipation by itself does not prevent electronic coherence from being observed. In spite of the above limitations, we hope that our results will encourrage experimental attempts to detect electronic coherence in the scenarios we have suggested.

\section{INITIAL PREPARATION EFFECTS FOR NONADIABATIC ELECTRON TRANSFER}

In this section, we briefly discuss the effect of nonstandard initial preparations in the nonadiabatic limit, $\Delta \ll \omega_{c}$. The effects should be smaller in this limit, because as argued above when the timescale for a electron hop, $1 / \Delta$, is much longer than the typical timescale of of the bath, $1 / \omega_{c}$, the initial bath state is not expected to matter. Studying the effects of shifted initial preparations in the nonadiabatic limit becomes especially interesting in the context of vibrational coherence, because a polarization distribution shifted away from the energy surface minimum may lead to an interesting dynamical behavior.

In the nonadiabatic limit, the ET transfer can be very accurately described in the framework of the noninteracting blip approximation (NIBA) [14]. Using the concept of the equivalent electric field, a nonstandard preparation can be easily incorporated in the NIBA. In fact, the proper form of the NIBA expression for $\dot{P}_{L}(t)$ in the presence of a driving field has already been given, e.g., in Refs. 38,41, though from a very different perspective. All we need to do is to modify these expressions by choosing the interaction energy $\Delta H(t)$ according to the external field in the form (15), getting

$$
\begin{aligned}
& \dot{P}_{L}(t)=-\left(\Delta^{2} / 2\right) \int_{0}^{t} d \tau e^{-S(\tau)}[\sin (R(\tau)) \times \\
& \sin (\epsilon \tau+(1+\bar{q})[R(t)-R(t-\tau)])+\cos (R(\tau)) \times \\
& \left.\cos (\epsilon \tau+(1+\bar{q})[R(t)-R(t-\tau)])\left(2 P_{L}(t-\tau)-1\right)\right] .
\end{aligned}
$$

We first examine the short time behavior of the ET. For times smaller than $1 / \omega_{c}, R(\tau)$ is proportional to the reorganization energy, $\lambda$, and at high temperatures, $S(\tau)=\lambda \tau^{2} / \hbar^{2} \beta$. With $P_{L}(t) \approx 1$ for $t \ll 1 / \omega_{c}$, one finds

$$
\Gamma(t) \equiv-\frac{\dot{P}_{L}(t)}{P_{L}(t)} \approx \int_{0}^{t} d \tau e^{-\lambda \tau^{2} / \hbar^{2} \beta} \cos ([(\lambda / \hbar) \bar{q}+\epsilon] \tau)
$$

The "rate" $\Gamma(t)$ defined here displays a few oscillations of frequency $[(\lambda / \hbar) \bar{q}+\epsilon]$ at extremely short times. This frequency corresponds to the vertical distance of the diabatic Marcus parabolas at $\mu \mathcal{E}=\bar{q} \lambda / 2$, so these oscillations are due to electronic resonance. In the nonadiabatic limit, the ET takes place on a much longer timescale compared to this resonance. As a result, although these oscillations can be observed in $P_{L}(t)$, they exist only for very short times (up to $\approx 1 / \omega_{c}$ ) and their amplitudes are only $1 \%$ of $P_{L}(t)$. Therefore, electronic coherence plays only a minor role in nonadiabatic ET.

The more interesting question is whether a nonstandard initial bath distribution causes effects on timescales $t \approx 1 / \omega_{c}$. Experiments in the photosynthetic reaction center have given evidence of vibrational coherence on the same timescale as the ET itself [1], where oscillations of the nuclear coordinate distribution (a "wavepacket") within the donor energy parabola could be observed before dephasing occurred. Since the nuclear coordinate $\mathcal{E}$ must lie in the Landau-Zener crossing region for $\mathrm{ET}$ to occur, the nuclear motions should result in oscillatory transfer rates and in the electronic occupation probabilities. As already pointed out, with an ohmic bath there is no vibrational coherence. However, one should still be able to detect the relaxation of the polarization from the transfer rate. To test this wavepacket picture, we chose to investigate a rather small electronic coupling, $\Delta=0.4 \omega_{c}$, with $\lambda=3 \hbar \omega_{c}$ and $\hbar \beta \omega_{c}=10$.

Figure 7 shows $P_{L}(t)$ for $\bar{q}=0,-1$ and -2 obtained from a NIBA calculation (solid lines), and from quantum dynamics simulations (dashed lines). $\bar{q}=-1$ corresponds to standard prepartion with the bath equilibrated to the donor state. $\bar{q}=0$ corresponds to a polarization distribution initially placed at the crossing point of the Marcus parabolas (inset of Fig. 7) where the majority of the ET occurs. The ET proceeds rapidly. Finally, $\bar{q}=-2$ corresponds to a polarization distribution centered on the opposite side of the minimum. To get to the crossing region, the bath has to first relax to the parabola minimum, causing a delay in the ET. This is most easily discerned from the rate $\Gamma(t)$. Figure 8 shows the difference of the rates for $\bar{q}=-2$ and 0 from the standard preparation $\bar{q}=-1$. They are clearly out of phase with each other, in agreement with the picture of two wavepackets starting at opposite turning points.

A different situation is found in the activationless regime, where the Landau-Zener crossing region is now at the bottom of the donor parabola. Two wavepackets starting at opposite turning points should result in rates that are in phase. Figure 9 shows that this is indeed the case for times up to $1 / \omega_{c}$. Once the polarization reaches the crossing region, the two situations are no longer symmetric. The inset of Fig. 9 shows a closeup of the crossing region. Compared to the one approaching from the right $(\bar{q}=4)$, the polarization coming from the left side $(\bar{q}=-6)$ will have an easier time tunneling, by simply sliding down the lower energy surface. This is reflected by the transfer rates shown in Fig. 9. We see that in the nonadiabatic regime nonstandard preparations also have a visible effect on the ET consistent with a simple wavepacket picture. However, the effect is much less significant than in the adiabatic regime.

Whether vibrational coherence causes oscillations in the populations or not depends on the specific spectral 
density that is involved. If the spectral density is dominated by a narrow range of frequencies, not surprisingly we find oscillations in $P_{L}(t)$ when a nonstandard initial preparation is used. These oscillations vanish when the polarization distribution is initially placed at the minimum of the donor parabola. The effect of vibrational coherence on the ET can be predicted by looking at the relaxation behavior of the initially shifted polarization distribution, i.e. the form of $\dot{R}(t)$ or the damping kernel. For the photosynthetic reaction center, the classical correlation functions have been determined by molecular dynamics simulations [24]. Although they show some high frequency oscillatory modulations, the basic behavior is an overdamped relaxation. We conclude that vibrational coherence should not cause significant oscillations in the electron populations in the reaction center, even if the initial wavepacket is largely displaced. This is also the conclusion from quantum dynamics simulations employing the spectral density determined in 24], and in agreement with recent experimental findings [42], while another group suggests the opposite 43].

Not surprisingly, taking a model spectral density containing a large concentration of weights within a very narrow range of frequencies, our NIBA and QMC calculations both showed that $P_{L}(t)$ becomes oscillatory.

\section{CONCLUDING REMARKS}

The unambiguous detectability of electronic coherence in ET reactions is still an open question from an experimental point of view. In this paper, we have shown that there are situations, in which one might be able to indeed observe oscillatory behaviors in electronic occupation probabilities which are caused by electronic coherence. The most appropriate systems seem to be symmetric mixed-valence compounds, which are close to the adiabatic ET regime. The crucial point is then to consider preparations where the solvent polarization initially forms a distribution centered near the LandauZener crossing region. We have given some examples for how one can create such an initial preparation in practice. Before the wavepacket slides away, i.e. before dephasing occurs, the electron can have enough time to oscillate back and forth several times between the electronic surfaces. The resulting large-amplitude oscillations should be easily detectable in experiments.

Besides the experimental relevance, we believe that these results might be of use for other theoretical studies of nonequilibrium preparations in condensed phase systems. We have extended the Feynman-Vernon influence functional method to account for nonstandard initial preparations.

From the path-integral expressions, one can easily deduce that such nonstandard preparations can be thought of as an equivalent external field. This establishes a link with the field of driven dissipative quantum systems and this connection is useful in actual computations. Another important consequence of this concept is that one can quite easily decide whether a particular ET system may exhibit oscillatory rates by inspecting the equivalent external field. If the latter does not show oscillations but simply relaxes to zero, the polarization motion will be overdamped, and oscillatory behaviors must have an electronic origin. For that reason, we conclude that vibrational coherence cannot cause oscillations in the occupation probabilities for a large class of common ET systems. A prominent example is the primary ET step in the photosynthetic reaction center.

To conclude, we hope that this paper will stimulate experiments investigating electronic coherence in electron transfer reactions, as well as theoretical studies dealing with the effects of special initial preparations, e.g. due to the laser pulse excitation of a wavepacket, in condensed phase systems.

\section{ACKNOWLEDGMENTS}

Throughout this work, we have benefitted from many enlightening discussions with Stephen Bradforth and Gerhard Stock. This research has been supported by the National Science Foundation under grant CHE-9528121 and by the Schwerpunkt "Zeitabhängige Phänomene und Methoden in Quantensystemen in der Physik und Chemie" of the Deutsche Forschungsgemeinschaft (DFG). CHM is a NSF Young Investigator (CHE9257094), a Camille and Henry Dreyfus Foundation Camille Teacher-Scholar and a Alfred P. Sloan Foundation Fellow. CHM acknowledges support from the DFG Schwerpunkt 276 during an extended stay at Freiburg. JA is a Feodor Lynen Fellow of the Humboldt Foundation. Computational resources have been provided by the IBM Corporation under the SUR Program at USC.

[1] M.H. Vos, J.-C. Lambry, S.J. Robles, D.C. Youvan, J. Breton and J.-L. Martin, Proc. Natl. Acad. Sci. U.S.A 88, 8885 (1991); M.H. Vos, M.R. Jones, C.N. Hunter, J. Breton, J.-C. Lambry and J.-L. Martin, Biochemistry 33, 6750 (1994); M.H. Vos, F. Rappaport, J.-C. Lambry, J. Breton and J.-L. Martin, Nature 363, 320 (1993).

[2] R.J. Stanley and S.G. Boxer, J. Phys. Chem. 99, 859 (1995).

[3] D.C. Arnett, P. Vöhringer and N.F. Scherer, J. Am. Chem. Soc. 117, 12262 (1995).

[4] G.G. Kochendorfer and R.A. Mathies, Israel J. of Chemistry 35, 211 (1996).

[5] M. Chachisvilis and V. Sundström, Chem. Phys. Lett. 261, 165 (1996). 
[6] K. Wynne, G.D. Reid and R.M. Hochstrasser, J. Chem. Phys. 105, 2287 (1996).

[7] P.J. Reid, C. Silva, P.F. Barbara, L. Karki and J.T. Hupp, J. Phys. Chem. 99, 2609 (1995).

[8] J.M. Jean, R.A. Friesner and G.R. Fleming, J. Chem. Phys. 96, 5827 (1992), J.M. Jean and G. R. Fleming, J. Chem. Phys. 103, 2092 (1995).

[9] R.G. Alden, W.D. Cheng and S.H. Lin, Chem. Phys. Lett. 194, 318 (1992), S.H. Lin, R.G. Alden, M. Hayashi, S. Suzuki and H.A. Murchison, J. Phys. Chem. 97, 12566 (1993).

[10] M. Ben-Nun, R.D. Levine and G.R. Fleming, J. Chem. Phys. 105, 3035 (1996).

[11] R.D. Coalson, D.G. Evans and A. Nitzan, J. Chem. Phys. 101, 436 (1994).

[12] D.G. Evans and R.D. Coalson, J. Chem. Phys. 104, 3598 (1995).

[13] C. Creutz, Prog. Inorg. Chem. 30, 1 (1983).

[14] A.J. Leggett, S. Chakravarty, A.T. Dorsey, M.P.A. Fisher, A. Garg and W. Zwerger, Rev. Mod. Phys. 59, 1 (1987).

[15] We note that bath modes with frequencies $\omega \gg \Delta$ can actually be incorporated by an adiabatic elimination procedure 14, 16], which simply leads to a renormalization of $\Delta$. In what follows, we imagine that such high-frequency modes have already been eliminated.

[16] X. Song and A.A. Stuchebrukhov, J. Chem. Phys. 99, 969 (1993); A.A. Stuchebrukhov and X. Song, J. Chem. Phys. 101, 9354 (1994).

[17] R.A. Marcus, J. Chem. Phys. 24, 996 (1956); R.A. Marcus and N. Sutin, Biochim. Biophys. Acta 811, 265 (1985).

[18] D. Chandler, Introduction to Modern Statistical Mechanics (Oxford University Press, 1987).

[19] D. Chandler, in Liquids, Freezing and Glass Transition, ed. by D. Levesque, J.P. Hansen and J. Zinn-Justin, (Elsevier Science Publishers, 1991).

[20] J.T. Stockburger and C.H. Mak, J. Chem. Phys. 105, 8126 (1996).

[21] U. Weiss, Quantum Dissipative Systems (World Scientific, Singapore, 1993).

[22] R.M. Stratt and M. Maroncelli, J. Phys. Chem. 100, 12981 (1996).

[23] S. Creighton, J.K. Hwang, A. Warshel, W.W. Parson and J. Norris, Biochemistry 27, 774 (1988); A. Warshel and W.W. Parson, Annu. Rev. Phys. Chem 42, 279 (1991).

[24] M. Marchi, J.N. Gehlen, D. Chandler and M. Newton, J. Am. Chem. Soc. 115, 4178 (1993).

[25] K. Schulten and M.Tesch, Chem. Phys. 158, 421 (1991).

[26] Note that the initial preparation specified by Eq. (8) does not actually yield the true thermal ET rate. The true thermal ET rate constant is related to the equilibrium time-correlation function of the projection operator $|L\rangle\langle L|$, which does not correspond to the factorized initial preparation in Eq. (\$). However, in most cases where the friction coefficient $\alpha$ is large, the resulting difference in the calculated ET rate is negligible.

[27] R.P. Feynman, Rev. Mod. Phys. 20, 367 (1948); R.P. Feynman and A.R. Hibbs, Quantum Mechanics and Path Integrals, Mc Graw-Hill, New York (1965).
[28] R.P. Feynman and F.L. Vernon, Ann. Phys. (N.Y.) 24, 18 (1963).

[29] D. Chandler and P.G. Wolynes, J. Chem. Phys. 74, 4078 (1981).

[30] C.H. Mak and D. Chandler, Phys. Rev. A 44, 2352 (1991).

[31] H. Grabert, P. Schramm and G.-L. Ingold, Phys. Rep. 168, 115 (1988).

[32] R. Egger, C.H. Mak and U. Weiss, Phys. Rev. E 50, R655 (1994).

[33] R. Egger and C.H. Mak, Phys. Rev. B 50, 15210 (1994).

[34] R. Egger and C.H. Mak, J. Phys. Chem. 98, 9903 (1994).

[35] C.H. Mak and R. Egger, Chem. Phys. Lett. 238, 149 (1995).

[36] C.H. Mak and R. Egger, Adv. Chem. Phys. Vol.XCIII, 39 (1996).

[37] C.H. Mak, in Encyclopedia of Computational Chemistry, ed. by P. Schleyer, N.L. Allinger, T. Clark, J. Gasteiger, P.A. Kollman and H.F. Schaefer III, (Wiley, New York, to appear 1997).

[38] M. Grifoni, M. Sassetti, J. Stockburger and U. Weiss, Phys. Rev. E 48, 3497 (1993); M. Grifoni, M. Sassetti, P. Hänggi and U. Weiss, ibid. 52, 3596 (1995); M. Grifoni, M. Sassetti and U. Weiss, ibid. 53, R2033 (1996).

[39] Yu. Dakhnovskii, Ann. Phys. (N.Y.) 230, 145 (1994).

[40] Yu. Dakhnovskii and R.D. Coalson, J. Chem. Phys. 103, 2908 (1995).

[41] D.G. Evans, R.D. Coalson, H.J. Kim and Yu. Dakhnovskii, Phys. Rev. Lett. 75, 3649 (1995).

[42] W. Zinth, private communication.

[43] A.M. Streltsov, T.J. Aartsma, A.J. Hoff and V.A. Shuvalov, Chem. Phys. Lett. 266, 347 (1997). 

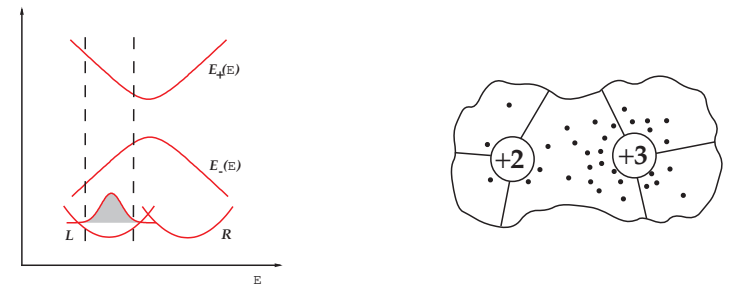

(a)

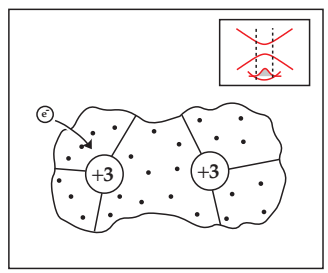

(b)

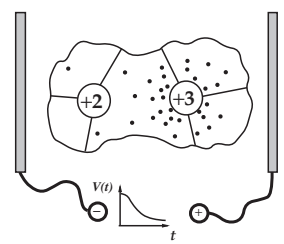

(d)

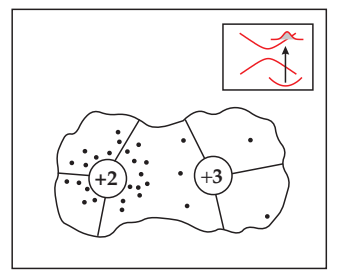

(c)

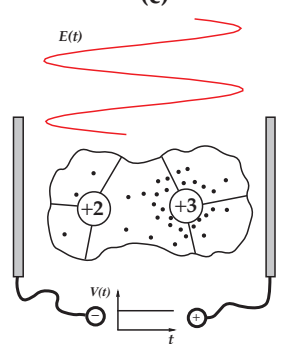

(e)
FIG. 1. (a) The electronic energy eigenvalues $E_{ \pm}(\mathcal{E})$ as a function of the bath polarization $\mathcal{E}$. The shaded gaussian represents $\rho_{\mathrm{S}}(\mathcal{E})$ that arises from a parabolic free energy surface (Marcus parabola). The mixed-valence compound for this standard preparation is sketched. The excess electron sits on the left metal center, and the bath polarization has adjusted to it (indicated by the density of dots). (b) Experimental situation where we expect large electronic coherence effects. For all times $t<0$, there is no electric dipole moment and therefore $\langle\mathcal{E}\rangle=0$, depicted by the uniform distribution of dots. At time $t=0$ an electron is injected into the left atom putting the electron in state $|L\rangle$, but with $\rho_{\mathrm{NS}}(\mathcal{E} ; \bar{q}=0, \beta)$ (see inset). (c) The initial preparation for backward ET in which the bath is equilibrated with respect to the excess electron in the acceptor. (d) While the actual system is prepared in the standard way, the initial bath polarization can be compensated by a suitable equivalent external field, so that the ET behaves just as in the case of Fig. 1(b). (e) In the actual experiment, it will be necessary to emulate the bath preparation using an optically produced equivalent external field atop a static background.

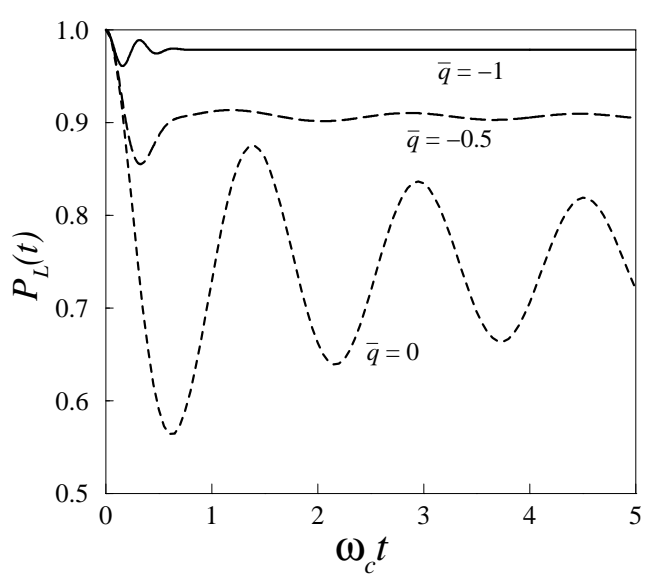

FIG. 2. $\quad P_{L}(t)$ evaluated in the adiabatic limit, for standard bath preparation $\rho_{\mathrm{S}}(\mathcal{E})$ (solid curve) and shifted bath preparations $\rho_{\mathrm{NS}}(\mathcal{E} ; \bar{q}=-0.5, \bar{\beta}=\beta)$ (long-dashed curve) and $\rho_{\mathrm{NS}}(\mathcal{E} ; \bar{q}=0, \bar{\beta}=\beta)$ (short-dashed curve). The parameters used were $\Delta=4 \omega_{c}, \lambda=20 \hbar \omega_{c}$ and $\hbar \beta \omega_{c}=3$ for a symmetric system, $\epsilon=0$. 

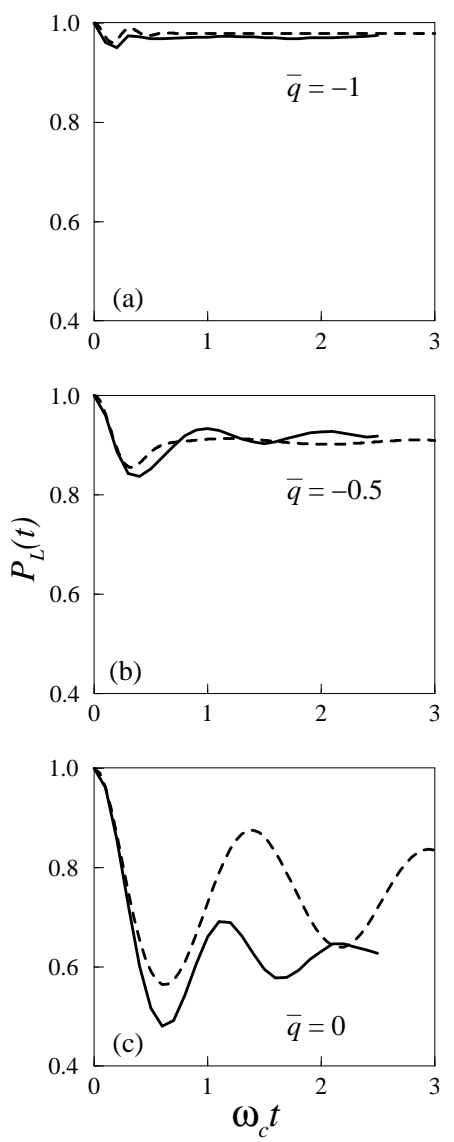

FIG. 3. Comparison of the data from Fig. 1 (dashed lines) with the corresponding QMC results (solid lines).

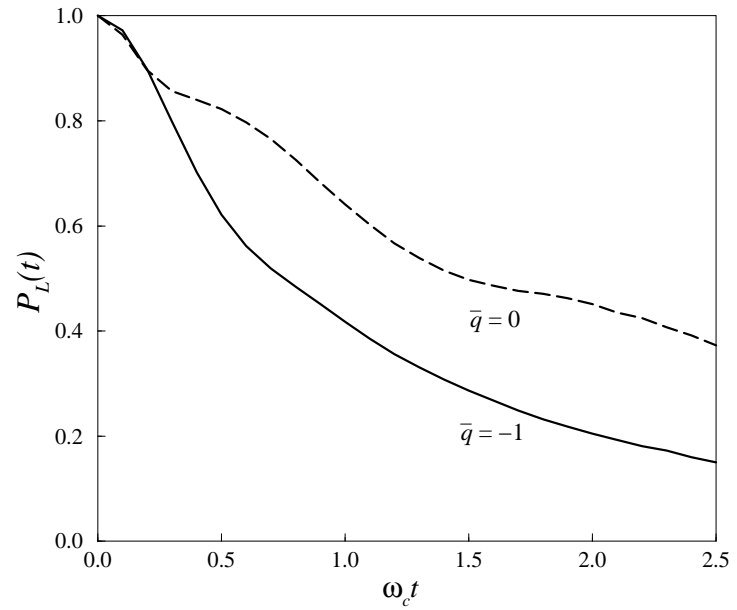

FIG. 4. QMC results for $P_{L}(t)$ in the activationless regime $\hbar \epsilon=\lambda$. Other parameters are the same as in Fig. 2 .

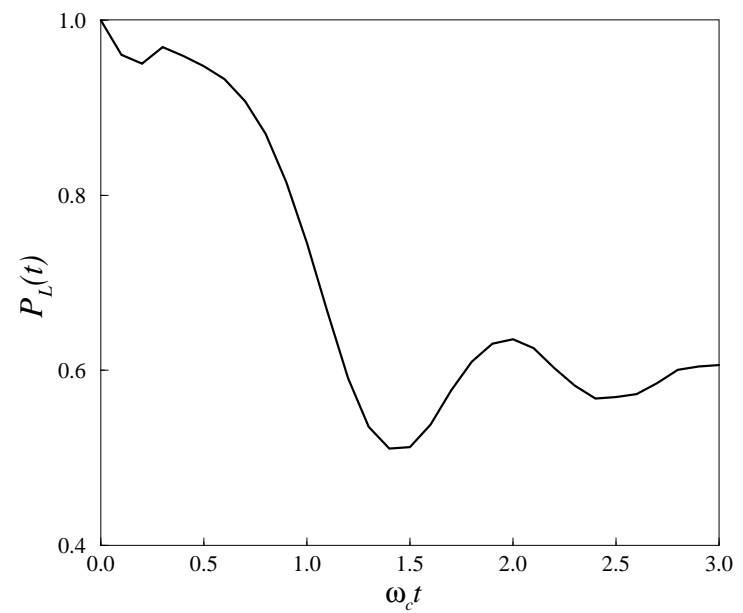

FIG. 5. QMC data for $P_{L}(t)$ in the case of a b-ET reaction. Parameters are the same as in Fig. 2. 


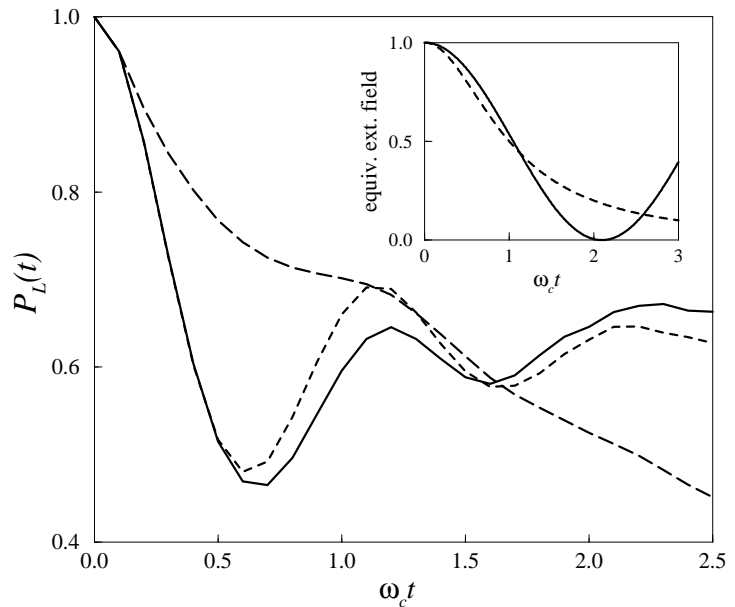

FIG. 6. $\quad P_{L}$ for the standard initial preparation but with an equivalent external field. In order to reproduce ET of the $\bar{q}=0$ initial preparation (short-dashed curve given here for comparison), the external field must take the form of the dashed line in the inset. If the equivalent field is approximated by a sinusoidal field on a static background (solid line in inset), the ET (solid line) behaves very similarly to the nonstandard preparation showing large electronic coherence. The long-dashed line is an estimate of the population if a random-phase cosine-modulated external field is used instead (see text).

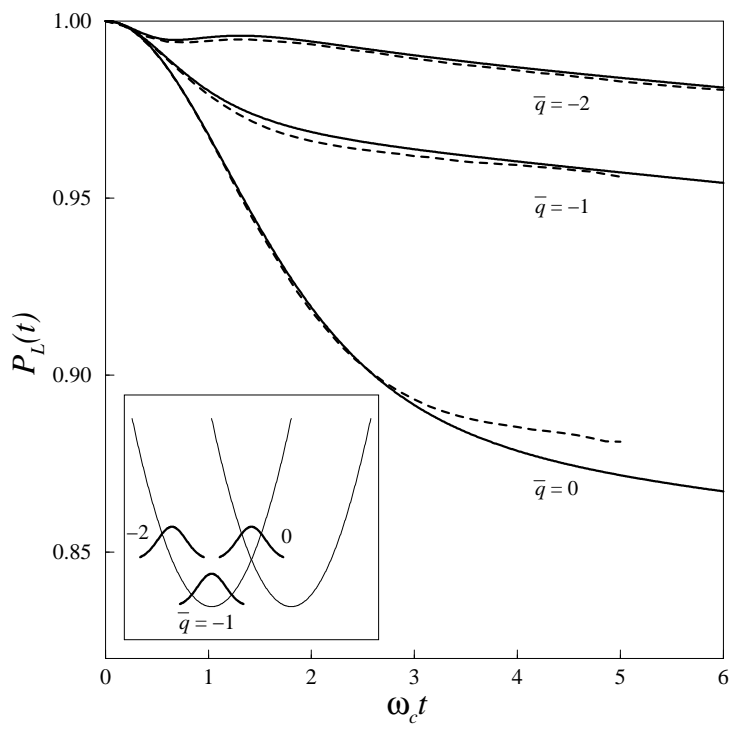

FIG. 7. $\quad P_{L}(t)$ for $\Delta=0.4 \omega_{c}, \lambda=3 \hbar \omega_{c}$ and $\hbar \beta \omega_{c}=10$ for initial bath preparations with $\bar{q}=0,-1,-2$ in the symmetric case $\epsilon=0$. The solid curves have been obtained in a NIBA calculation, the short-dashed curves by QMC. The inset shows the position of the corresponding initial polarization distributions in the Marcus picture.

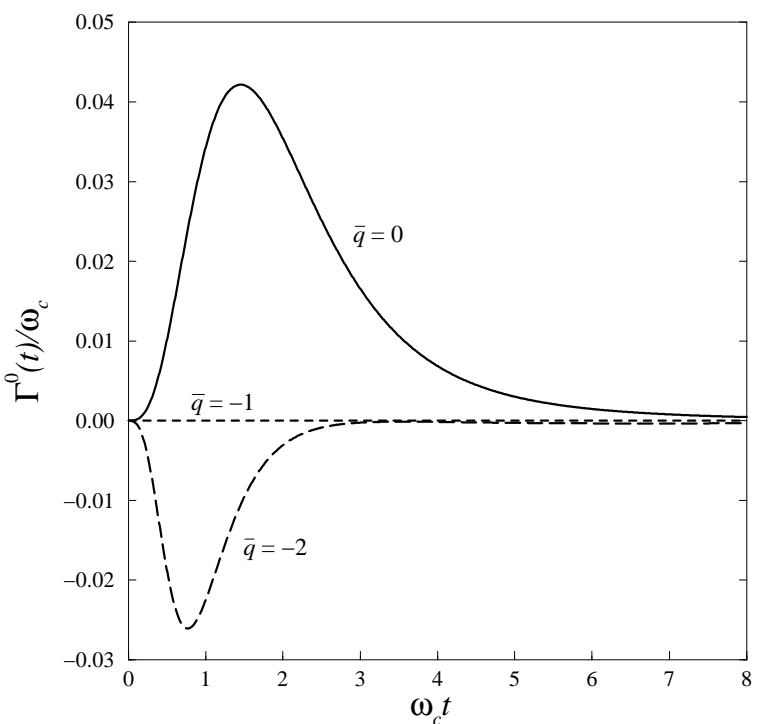


FIG. 8. The normalized transfer rates $\Gamma^{0}(t)$ for the situation of Fig. 7.

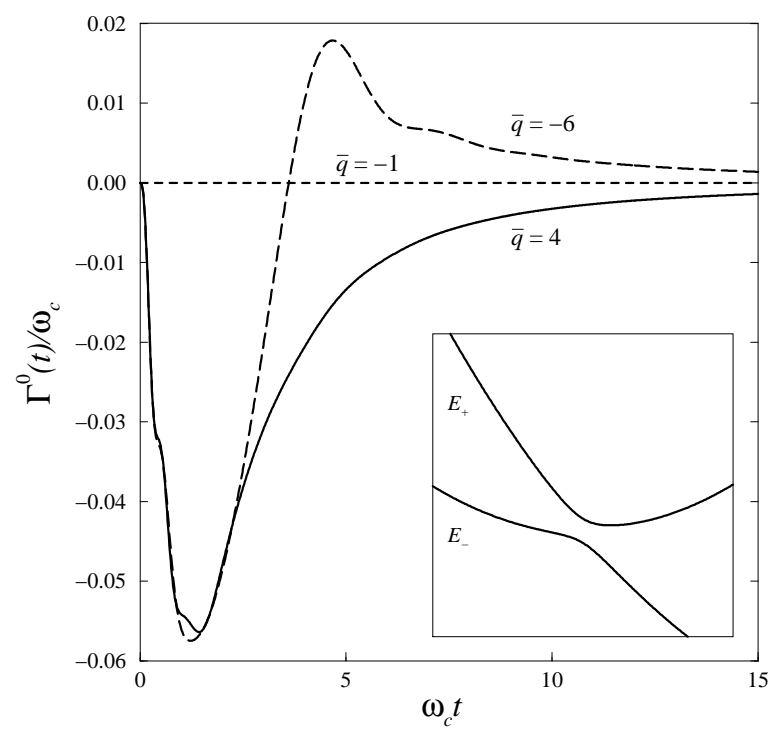

FIG. 9. The NIBA-calculated normalized transfer rates in the activationless regime $\hbar \epsilon=\lambda$. The wavepackets start at opposite sides of the donator parabola minimum. The inset shows a closeup look of the Landau-Zener crossing region, which allows to explain the asymmetric behavior in the wavepacket picture. 